\title{
First Cryogenic Testing of the ATLAS Superconducting Prototype Magnets
}

\author{
N. Delruelle, A. Dudarev, F. Haug, C. Mayri, J.-P. Orlic, G. Passardi, O. Pirotte, and H. ten Kate
}

\begin{abstract}
The superconducting magnet system of the ATLAS detector will consist of a central solenoid, two end-cap toroids and the barrel toroid made of eight coils (BT) symmetrically placed around the central axis of the detector. All these magnets will be individually tested in an experimental area prior to their final installation in the underground cavern of the LHC collider. A dedicated cryogenic test facility has been designed and built for this purpose. It mainly consists of a $1200 \mathrm{~W}$ at $4.5 \mathrm{~K}$ refrigerator, a $10 \mathrm{~kW}$ liquid nitrogen pre-cooling unit, a cryostat housing liquid helium centrifugal pumps, a distribution valve box and transfer lines. Prior to the start of the series tests of the BT magnets, two model coils are used at this facility. The first one, the so-called B00 of comparatively small size, contains the three different types of superconductors used for the ATLAS magnets which are wound on a cylindrical mandrel. The second magnet, the BO, is a reduced model of basically identical design concept as the final BT magnets. Full commissioning of all sub-systems including cryogenics, electrical powering, magnet protection and control is done ahead of the arrival of the BT series magnets. This cryogenic test facility reproduces the final thermo-hydraulic conditions in the cooling circuits of the magnets in order to validate the design concept.
\end{abstract}

Index Terms-ATLAS cryogenics, helium refrigeration, superconducting magnets.

\section{INTRODUCTION}

A TLAS will be the largest of the four detectors exploiting the capabilities of the CERN Large Hadron Collider (LHC) in high energy physics experiments colliding protons and heavy ions.

The magnetic field configuration of ATLAS [1] is based on four superconducting magnets, namely the Barrel Toroid (BT) built up of eight identical rectangular shaped "racetrack" coils, two identical End Caps Toroids (ECT) and the Central Solenoid (CS). This solenoid generates a longitudinal and uniform magnetic field for the inner tracker, whilst the BT and the two ECTs produce a toroidal field around the central axis for the muon spectrometer.

For testing two reduced-size prototype magnets and the eight BT coils prior to their underground final assembly, a dedicated cryogenic test facility has been set-up in a $5000 \mathrm{~m}^{2}$ experimental

Manuscript received September 24, 2001.

N. Delruelle, F. Haug, J.-P. Orlic, G. Passardi, and O. Pirotte are with CERN, LHC Division, 1211 Geneva 23, Switzerland (e-mail: \{Nicolas.Delruelle; Friedrich.Haug; Jean-Pierre.Orlic; Giorgio.Passardi; Olivier.Pirotte\} @ cern.ch).

A. Dudarev and H. ten Kate are with CERN, EP Division, 1211 Geneva 23, Switzerland (e-mail: Alexey.Dudarev@cern.ch).

C. Mayri is with CEA-Saclay, DAPNIA, 91191 Gif-sur-Yvette, France (e-mail: Mayri@dapnia.cea.fr).

Publisher Item Identifier S 1051-8223(02)04192-1. area [2]. This test facility consists of a helium refrigerator with an entropy-equivalent cooling power of $1200 \mathrm{~W}$ at $4.5 \mathrm{~K}$, a liquid nitrogen precooling unit of $10 \mathrm{~kW}$ in the range of 300 $\mathrm{K}-100 \mathrm{~K}$, a cryostat housing two immersed liquid helium centrifugal pumps, a distribution valve box and two transfer lines feeding the two test benches. A simplified process flow diagram is presented in Fig. 1.

The two prototype magnets that have been tested up to now, had two different purposes. The first magnet, the so-called B00 [3], consists of a coil wound on a cylindrical mandrel and made of the three different types of superconductors that will be used on BTs, ECTs and CS. The B00 tests allowed, in addition to the performance measurements of these superconductors, to check the correct operation of all sub-systems, namely the cryogenics, the electrical powering including the final $20.5 \mathrm{kA}$ current leads and the magnet protection/control systems prior to the test of the B0 and the final eight BT coils. The second magnet, called $\mathrm{B} 0$, is a prototype model with a 1/3 length of the final BTs and has a cold mass of 25 tons. The B0 was constructed in order to validate the design concept of the BTs.

This paper describes the cryogenic operation and presents the results obtained during the cool-down phase, the functioning of the pump cryostat interfacing the refrigerator with the magnet, the energy dump of the magnet and, finally, the warm-up phase.

\section{CRYOGENIC OPERATION}

The ATLAS toroid magnets will be indirectly cooled by helium flowing into pipes fixed to the cold mass. During cooldown, forced flow of high-pressure gaseous helium will be used, whereas two-phase forced flow of helium at $4.5 \mathrm{~K}$ will be required to keep the magnets at their operating temperature [4].

The B0 cold mass and thermal shield were cooled down from $300 \mathrm{~K}$ in parallel by the $1200 \mathrm{~W}$ refrigerator. The liquid nitrogen precooling unit was not used since the turbines cooling capacity was sufficient. This procedure was carried out in an automatic mode, maintaining a temperature gradient in the cold mass of $30 \mathrm{~K}$, and a cooling speed of about $1 \mathrm{~K} / \mathrm{h}$. Slow adaptation of the refrigerator cooling capacity was done by throttling the turbines inlet circuit, whereas fast response of the system was achieved by controlling the mass flow supplied to the magnet. Protection against overcooling, producing excessive temperature gradients, was guaranteed by mixing, when necessary, high-pressure gaseous helium at $300 \mathrm{~K}$ directly into the cold gas entering into the magnet. Final magnet cool-down $(20 \mathrm{~K}$ to $4.5 \mathrm{~K}$ ) was achieved by supplying, via the refrigerator, supercritical helium at $4.5 \mathrm{~K}$ which was expanded into the magnet. Fig. 2 presents the time evolution, during the two-week cool-down phase, of the B0 coil temperatures near the magnet helium inlet and outlet. 


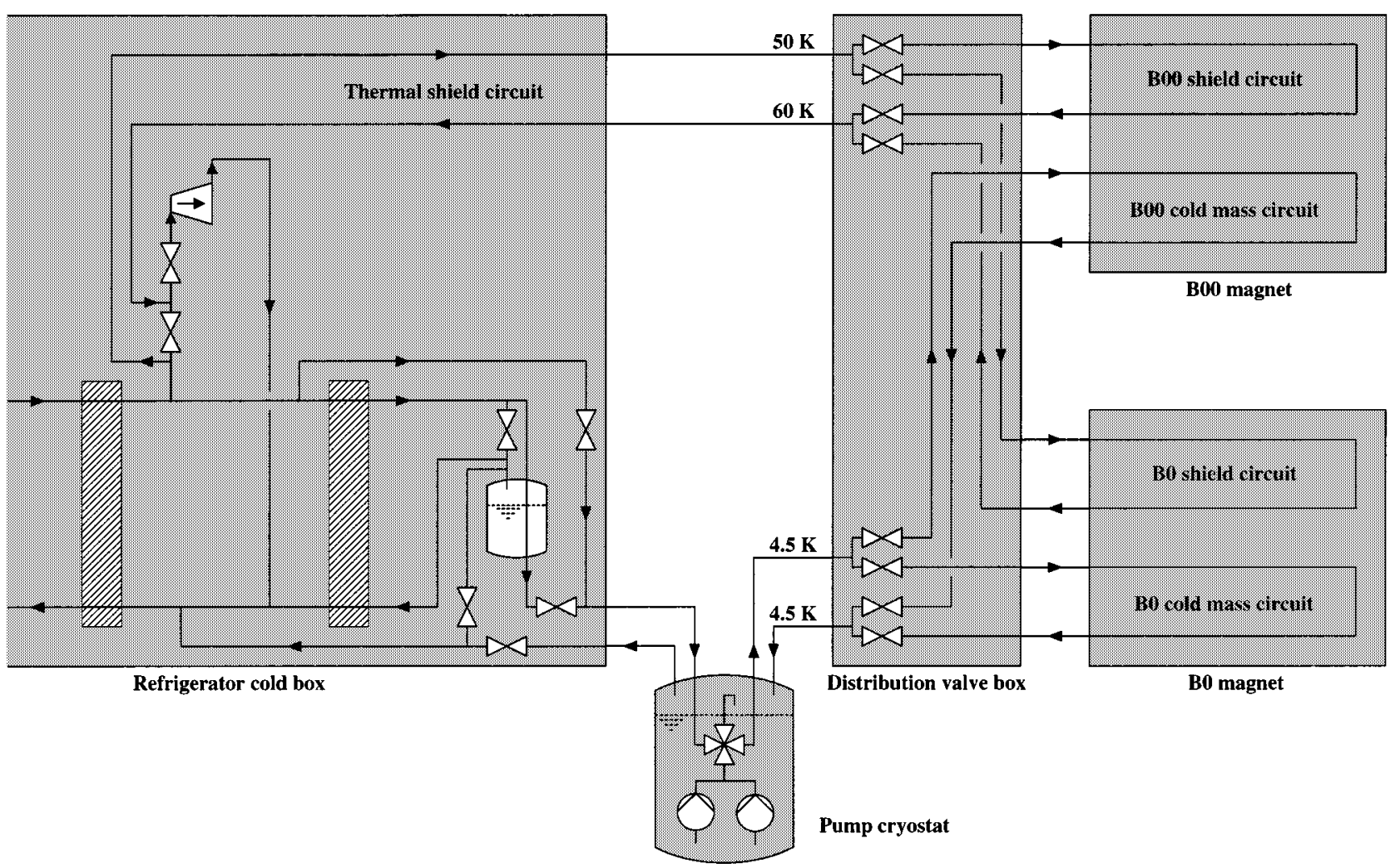

Fig. 1. Simplified process flow diagram of the cryogenic plant.

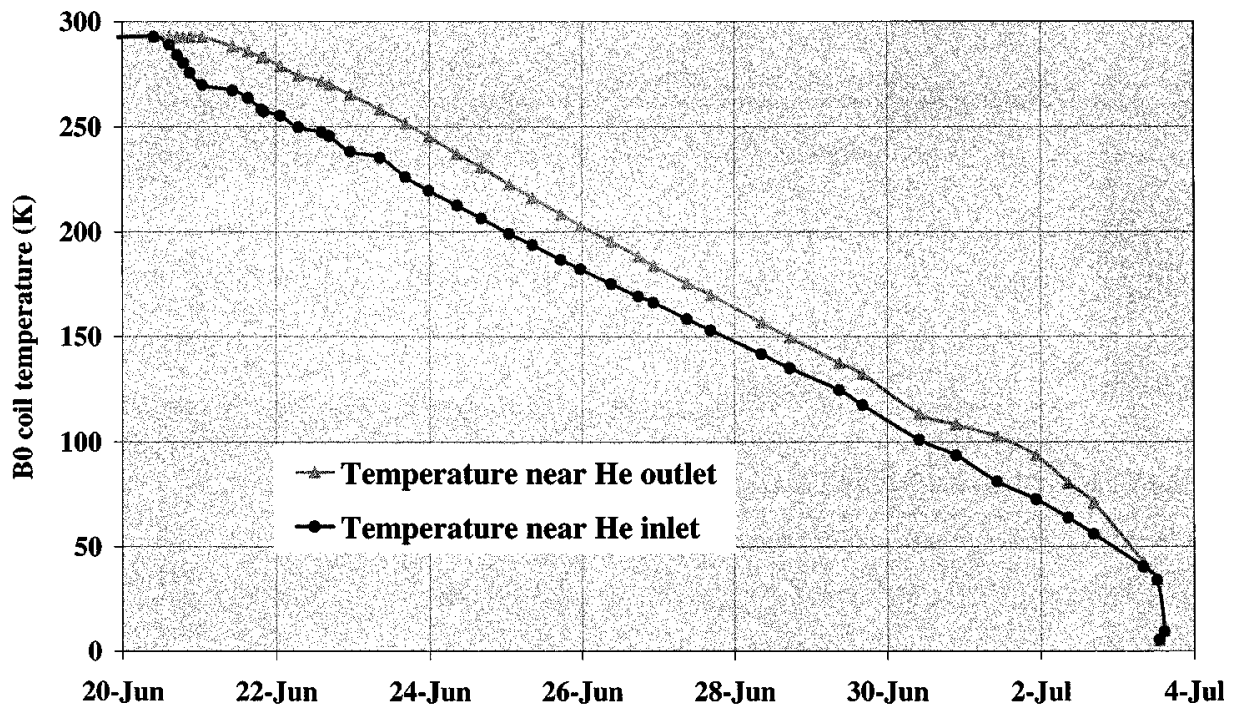

Fig. 2. Time evolution of the B0 coil temperatures during cool-down.

At the end of this cool-down, two-phase flow conditions were established inside the magnet circuit and the excess of liquid returning from the magnet was used to fill up the pump cryostat. Once the liquid helium level in the cryostat was sufficient, one of the two centrifugal pumps was started to circulate the helium into the magnet cold mass. The set point of the pump speed was not fixed but was a function of the cryostat level to provide a smooth start-up and avoid any surging of the pump. During this time, the refrigerator completed the cryostat filling and maintained afterwards its level to a constant value. In case of an unexpected stop of the cryoplant, the cryostat houses $500 \mathrm{~L}$ of liquid helium, a quantity which allows, with regard to the total heat load of the system, to continue the magnet cooling for more than 90 minutes. When the pump cryostat was in steady-state conditions, the cryogenics authorized the magnet control system to ramp-up the current to its nominal value of $20.5 \mathrm{kA}$. As long as the magnet was energized (actually, when its current was greater than $1 \mathrm{kA}$ ) the set point of the pump speed was no longer function of the liquid helium level but remained constant.

During the cool-down of the magnet, the current leads receive a constant flow of $2 \times 0.45 \mathrm{~g} / \mathrm{s}$ withdrawn directly from the cold mass cooling circuit. When the magnet is energized, the cooling 


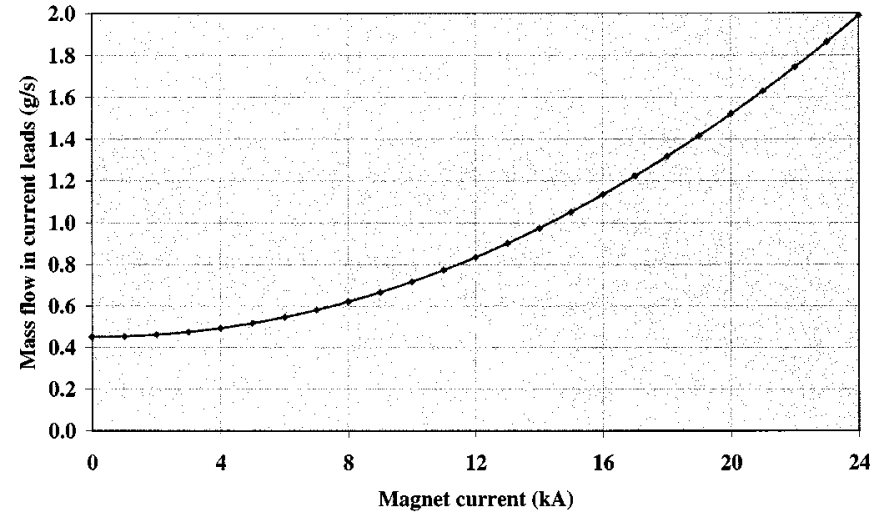

Fig. 3. Cooling mass flow in current leads versus magnet current.

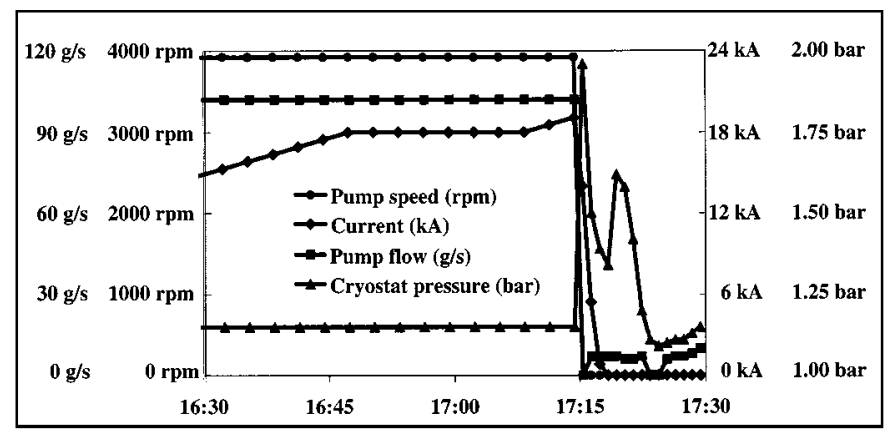

Fig. 4. Cryostat parameters versus time after a quench at $19 \mathrm{kA}$.

of the current leads is controlled by two PID loops in cascade. A fast loop regulates, via a control valve, a predefined helium mass flow which is a function of the current injected into the coil, while a second slower loop controls the helium outlet temperature of the current leads to maintain permanently a temperature slightly below ambient $(\approx 270 \mathrm{~K})$. Correlation between the current leads cooling and the magnet current is shown in Fig. 3.

Tests showed that slow energy dumps of the magnet do not affect significantly the operating conditions of the cryoplant which could therefore remain in its baseline mode. During a fast energy dump, however, the total stored energy (46 MJ) is discharged into the cold mass by resistive transition of the coil induced by four quench heaters of $300 \mathrm{~W}$ each. The pump is then stopped and all valves at the interface between the refrigerator and the cryostat are closed. This prevents the cryoplant from stopping due to an excessive compressor suction pressure produced by the sudden discharge of the $18 \mathrm{~L}$ of liquid helium contained in the magnet circuit. After a quench at $19 \mathrm{kA}$, the pump cryostat pressure increased in one minute from its nominal value of 1.2 bar abs. to a peak value of almost 2 bar abs. (see Fig. 4).

For the warm-up of the B0, direct electrical heating through the windings has been chosen for the cold mass, whereas forced flow of high-pressure gaseous helium provided by the refrigerator was used for the thermal shields. The warm-up speed of the cold mass was identical to the cooling one, i.e., $1 \mathrm{~K} / \mathrm{h}$.

\section{RESULTS}

The centrifugal pumps were operated at their nominal speed of $3950 \mathrm{rpm}$, providing a mass flow of $100 \mathrm{~g} / \mathrm{s}$, with a measured overall hydraulic impedance of the cryogenic circuit of about 275 mbar. As the B0 magnet is equipped with 8 parallel cooling circuits, each of them with an inner diameter of $14 \mathrm{~mm}$, these $100 \mathrm{~g} / \mathrm{s}$ are divided up among the 8 cooling circuits to give, in each circuit, a mass flow rate per unit area of $8.1 \mathrm{~g} . \mathrm{s}^{-1} . \mathrm{cm}^{-2}$, a value twice the minimum of $4 \mathrm{~g} . \mathrm{s}^{-1} . \mathrm{cm}^{-2}$ requested to ensure the hydraulic stability [4].

In steady-state conditions, the B0 thermal shield temperature was kept between $50 \mathrm{~K}$ at the inlet and $60 \mathrm{~K}$ at the outlet.

The current leads operation at nominal current of $20.5 \mathrm{kA}$ required $2 \times 1.55 \mathrm{~g} / \mathrm{s}$ of helium cooling flow.

Cool-down from $300 \mathrm{~K}$ of the B0 magnet was achieved in two weeks and automatic switching from final cool-down mode, where helium flow is forced by the refrigerator, to the baseline operation, where the pump is running, has been done successfully.

Very stable cryogenic conditions were obtained in the baseline mode. The operation of the whole cryoplant was only slightly affected by a slow energy dump of the magnet, whereas a fast energy dump imposed rapid isolation of the refrigerator from the pump cryostat to protect it from the resulting backing pressure.

Warm-up of the cold mass by direct electrical heating through the windings did not create any problem.

Precise thermal load measurements of the B0 magnet could not be carried out during this preliminary tests and are planned for the near future.

\section{CONCLUSIONS}

The cryogenic design concept of indirect cooling by twophase helium forced-flow provided by a centrifugal pump has been validated.

The B0 cool-down procedure, with its related thermal constraints, and the warm-up method of the cold mass, consisting of direct electrical heating through the windings, were successfully tested.

All sub-systems such as cryogenics, electrical powering including the final current leads, magnet protection and control systems have been commissioned. Minor changes have to be implemented in order to be ready for the "series" testing of the eight Barrel Toroid coils.

\section{ACKNOWLEDGMENT}

The authors would like to give special thanks to G. Cuccuru, Y. Drouyer, S. Junker, D. Legrand, J. Metselaar and A. Park who provide valuable technical assistance during the construction, commissioning and operation of this test facility.

\section{REFERENCES}

[1] ATLAS Magnet System: Technical Design Report, Apr. 1997.

[2] F. Haug, A. Cambon, N. Delruelle, J-P. Orlic, G. Passardi, and J. Tischhauser, "The CERN cryogenic test facility for the ATLAS barrel toroid magnets," MT-16, Ponte Vedra Beach, USA, Sept. 1999.

[3] A. Dudarev, E. Boxman, H. ten Kate, O. Anashkin, V. Keilin, and V. Lysenko, "The B00 model coil in the ATLAS magnet test facility," IEEE Trans. Appl. Superconduct., vol. 11, no. 1, Mar. 2001.

[4] N. Delruelle, F. Haug, G. Passardi, and H. Ten Kate, "The helium cryogenic system for the ATLAS experiment," MT-16, Ponte Vedra Beach, USA, Sept. 1999. 